\title{
Excess $\mathrm{GeV}$ radiation and cosmic ray origin
}

\author{
I. Büsching, M. Pohl, and R. Schlickeiser
}

\author{
Institut für Theoretische Physik, Lehrstuhl IV: Weltraum- und Astrophysik, Ruhr-Universität Bochum, \\ 44780 Bochum, Germany \\ e-mail: mkp@tp4.ruhr-uni-bochum.de; rsch@tp4.ruhr-uni-bochum.de
}

Received 21 March 2001 / Accepted 8 August 2001

\begin{abstract}
Particle acceleration at supernova remnant (SNR) shock waves is regarded as the most probable mechanism for providing Galactic cosmic rays at energies below $10^{15} \mathrm{eV}$. The Galactic cosmic ray hadron component would in this picture result from the injection of relativistic particles from many SNRs. It is well known that the superposition of individual power law source spectra with dispersion in the spectral index value, which behaviour is observed in the synchrotron radio spectra of shell SNR, displays a positive curvature in the total spectrum and in particular shows a hardening at higher energies. Recent observations made with the EGRET instrument on the Compton Gamma-Ray Observatory of the diffuse Galactic $\gamma$-ray emission reveal a spectrum which is incompatible with the assumption that the cosmic ray spectra measured locally hold throughout the Galaxy: the spectrum above $1 \mathrm{GeV}$, where the emission is supposedly dominated by $\pi^{0}$-decay, is harder than that derived from the local cosmic ray proton spectrum. We demonstrate that in case of a SNR origin of cosmic ray nucleons part of this $\gamma$-ray excess may be attributed to the dispersion of the spectral indices in these objects. In global averages, as are $\gamma$-ray line-of-sight integrals, this dispersion leads to a positive curvature in the composite spectrum, and hence to modified $\pi^{0}$-decay $\gamma$-ray spectra.
\end{abstract}

Key words. ISM: cosmic rays - ISM: supernova remnants - gamma rays: theory

\section{Introduction}

Particle acceleration at supernova remnant shock waves is regarded as the most probable mechanism for providing Galactic cosmic rays at energies below $10^{15} \mathrm{eV}$ (for a review see Blandford \& Eichler 1987). The recent detections of non-thermal X-ray synchrotron radiation from the four supernova remnants SN1006 (Koyama et al.1995), RX J1713.7-3946 (Koyama et al. 1997; Slane et al. 1999), Cas A (Allen et al. 1997), and RCW86 (Borkowski et al. 2001) and the subsequent detections of SN1006 (Tanimori et al. 1998) and RX J1713.7-3946 (Muraishi et al. 2000) at $\mathrm{TeV}$ energies support the hypothesis that at least Galactic cosmic ray electrons are accelerated predominantly in SNR. To date, there is still no unambiguous proof that cosmic ray nucleons are similarly produced in SNR.

Whatever the nature of their sources, upon escape from there the cosmic ray nucleons would diffusively propagate through the interstellar medium, where they can be either directly measured or indirectly traced by means of $\gamma$-ray observations. Recent observations made with the EGRET instrument on the Compton Gamma-Ray Observatory of the diffuse Galactic $\gamma$-ray emission reveal a spectrum which is incompatible with the assumption that

Send offprint requests to: I. Büsching,

e-mail: ib@tp4.ruhr-uni-bochum.de the cosmic ray spectra measured locally hold throughout the Galaxy (Hunter et al. 1997). The spectrum observed with EGRET below $1 \mathrm{GeV}$ is in accord with, and supports, the assumption that the cosmic ray spectra and the electron-to-proton ratio observed locally are uniform, however, the spectrum above $1 \mathrm{GeV}$, where the emission is supposedly dominated by $\pi^{0}$-decay, is harder than that derived from the local cosmic ray proton spectrum.

In a recent paper Pohl \& Esposito (1998) demonstrated that if the sources of cosmic rays are discrete, as are SNR, then the spectra of cosmic ray electrons would vary and the locally measured electron spectrum would not be representative for the electron spectra elsewhere in the Galaxy, which could be substantially harder than the local one. These authors have shown that the observed excess of $\gamma$-ray emission above $1 \mathrm{GeV}$ can in fact be explained as a correspondingly hard inverse Compton component, provided the bulk of cosmic ray electrons is produced in SNR.

In the following we will demonstrate that in case of a SNR origin of cosmic ray nucleons part of the $\gamma$-ray excess may also be attributed to the dispersion of the spectral indices in these objects. In global averages, as are $\gamma$-ray line-of-sight integrals, this dispersion leads to a positive curvature in the composite spectrum, and hence to modified $\pi^{0}$-decay $\gamma$-ray spectra. 


\section{The dispersion of cosmic ray spectra in SNR}

The synchrotron radio spectra from shell SNR indicate power law spectra $I_{\nu} \propto \nu^{-\alpha}$ (Clark \& Caswell 1976; Milne 1979; Green 2001) where the spectral index distribution from the sample of shell supernova remnants (SNR) has a mean value of about $\langle\alpha\rangle \simeq 0.5$ and a significant standard deviation $\sigma_{\alpha}$. From synchrotron radiation theory this implies a power law energy distribution of the radiating relativistic electrons with mean spectral index $s_{\mathrm{e}}=1+2\langle\alpha\rangle \simeq 2.0$ and dispersion $\sigma_{\mathrm{e}}=2 \sigma_{\alpha}$. Because the age of the SNRs is much smaller than the characteristic radiative loss times of both cosmic ray nucleons and $\mathrm{GeV}$ electrons in the remnant, and because both the particle acceleration processes and the spatial propagation scale with rigidity, the relativistic hadrons should have the same momentum spectrum as the relativistic electrons. After leaving their source these particles propagate in the interstellar medium by momentum-dependent spatial diffusion with a diffusion coefficient $\kappa \propto p^{b}$, with $b \simeq 0.6$ as inferred from the measurement of secondary to primary cosmic ray elements. Consequently, the source spectrum of cosmic ray hadrons

$N(p)=N_{0}\left(\frac{p}{m c}\right)^{-s}$

is a power law in momentum per nucleon with a spectral index having a mean value $\langle s\rangle=1+b+2\langle\alpha\rangle \simeq 2.7$ and dispersion $\sigma=\sigma_{\mathrm{e}}$.

The Galactic cosmic ray hadron component results from the injection of relativistic particles from many SNRs, especially in the inner part of the Galaxy. In discussing the pros and cons of the Galactic origin of cosmic rays, Brecher \& Burbidge (1972) noted that the superposition of individual power law source spectra with dispersion in the spectral index value displays a positive curvature in the total cosmic ray spectrum and in particular shows a flattening at higher energies, simply because those sources with the smallest spectral index dominate the total spectrum at large energies. If we represent the distribution of hadron spectral indices by the Gaussian

$n(s)=\frac{1}{\sqrt{2 \pi} \sigma} \exp \left[-\frac{(s-<s>)^{2}}{2 \sigma^{2}}\right]$

we obtain from Eq. (1) for the averaged hadron spectrum

$$
\begin{aligned}
<N(p)> & =\int_{-\infty}^{\infty} \mathrm{d} s N(p, s) n(s) \\
& =N_{0}\left(\frac{p}{m c}\right)^{-<s>+\frac{\sigma^{2}}{2} \ln \left(\frac{p}{m c}\right)} .
\end{aligned}
$$

By using $N(p) \mathrm{d} p=N(\gamma) \mathrm{d} \gamma$ and $p=m c \sqrt{\gamma^{2}-1}$ we can determine from Eq. (3) the corresponding differential hadron spectrum with respect to the hadron Lorentz factor as

$N(\gamma)=N_{0} m c \gamma\left(\gamma^{2}-1\right)^{\frac{-<s>-1}{2}+\frac{\sigma^{2}}{8} \ln \left(\gamma^{2}-1\right) .}$
With increasing hadron Lorentz factor $\gamma$ the energy spectrum (3) flattens, i.e. it hardens. Calculating the slope yields

$$
\begin{aligned}
\bar{s} & \equiv-\frac{\ln <N(\gamma)>}{\ln \gamma} \\
& =\frac{<s>+1}{2} \frac{\ln \left(\gamma^{2}-1\right)}{\ln \gamma}-1-\frac{\sigma^{2}}{8} \frac{\ln ^{2}\left(\gamma^{2}-1\right)}{\ln \gamma} .
\end{aligned}
$$

At relativistic energies Eq. (5) approaches

$\bar{s} \simeq<s>-\frac{\sigma^{2}}{2} \ln \gamma$

\section{Pion decay gamma rays}

For the $\pi^{0} \rightarrow 2 \gamma$ decay the omnidirectional (i.e. integrated over the whole solid angle) differential $\gamma$-ray source function $q_{\pi^{0}}\left(E_{\gamma} ; \boldsymbol{r}\right)$ at the position $\boldsymbol{r}=(l, b, r)$ in space is related to the omnidirectional differential neutral pion source function

$q_{\pi^{0}}\left(E_{\gamma} ; \boldsymbol{r}\right)=2 \int_{U\left(E_{\gamma}\right)}^{\infty} \mathrm{d} \gamma_{\pi} \frac{Q_{\pi}\left(\gamma_{\pi} ; \boldsymbol{r}\right)}{\left[\gamma_{\pi}^{2}-1\right]^{1 / 2}}$

with the lower integration boundary

$U\left(E_{\gamma}\right)=\frac{E_{\gamma}}{m_{\pi} c^{2}}+\frac{m_{\pi} c^{2}}{4 E_{\gamma}}=f+\frac{1}{4 f}$

in terms of the dimensionless $\gamma$-ray photon energy

$f \equiv \frac{E_{\gamma}}{m_{\pi} c^{2}}=\frac{E_{\gamma}}{0.135 \mathrm{GeV}}$

in units of the pion rest energy.

The differential photon number flux of $\pi^{0}$-decay $\gamma$-rays from a direction $(l, b)$ is given by the line of sight integral of the source Eq. (7)

$\frac{\mathrm{d} N_{\gamma}\left(E_{\gamma} ; l, b\right)}{\mathrm{d} t \mathrm{~d} E_{\gamma} \mathrm{d} \Omega}=\frac{1}{4 \pi} \int_{0}^{\infty} \mathrm{d} r q_{\pi^{0}}\left(E_{\gamma} ; \boldsymbol{r}\right)$.

\subsection{Pion source spectrum}

The pion source spectrum has been frequently calculated on the basis of the inclusive cross section and a delta-functional for the pion spectrum (e.g. Mannheim \& Schlickeiser 1994). As we show in the Appendix this approximation may be useful at higher energies, but it is certainly inappropriate at energies not very far from the threshold.

Therefore in this section the pion source spectrum required in Eq. (7) is calculated using cosmic hadron distribution function (3) as input for the Monte-Carlo code DTUNUC (V2.2) (Möhring \& Ranft 1991; Ranft et al. 1994; Ferrari et al. 1996a; Engel et al. 1997), which is based on a dual parton model (Capella et al. 1994). This MC model for hadron-nucleus and nucleus-nucleus interactions includes various modern aspects of high-energy physics and has been successfully applied to the description of hadron production in high-energy collisions 


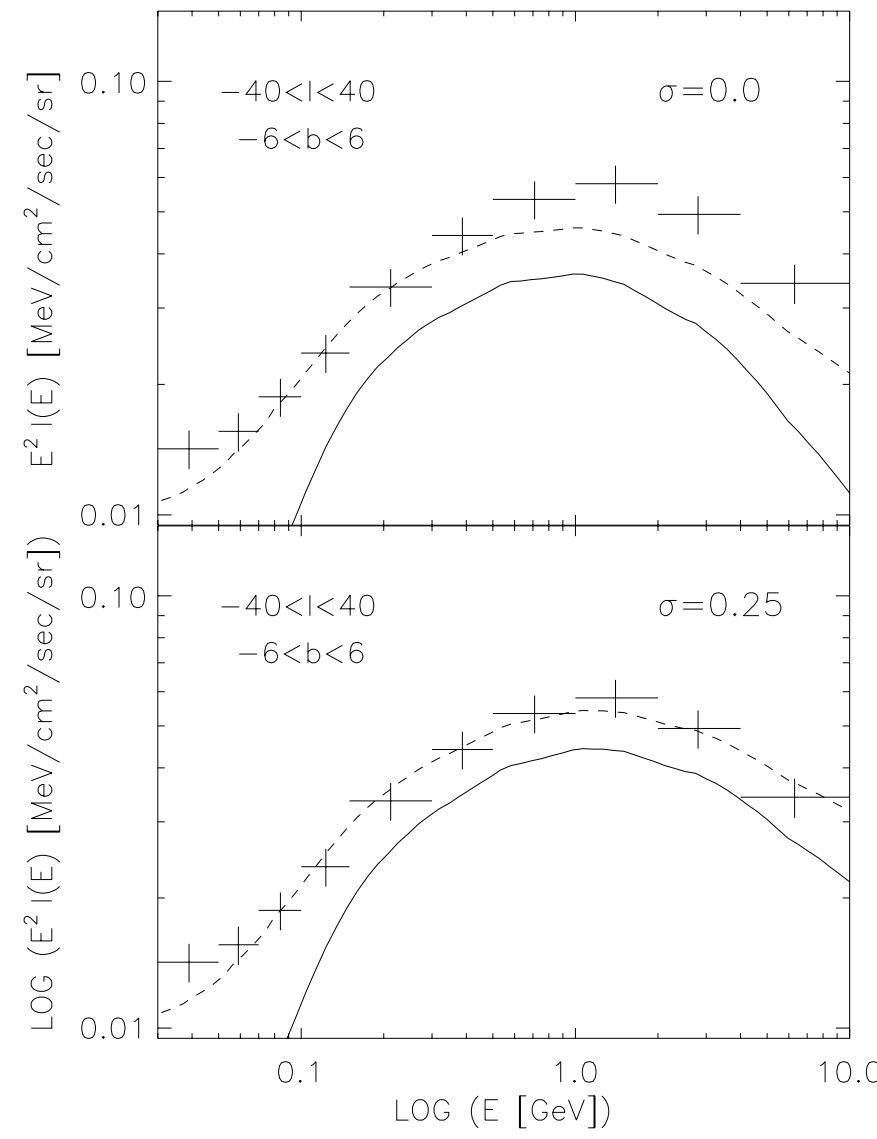

Fig. 1. The observed intensity spectrum from the inner Galaxy shown in comparison with $\pi^{0}$-decay spectra with (bottom panel) and without (top panel) dispersion in the cosmic ray spectrum. The solid lines display the $\pi^{0}$-decay spectra, and the dashed lines are the total $\gamma$-ray spectra including the leptonic contribution, which here is simply given as a power law $\propto E^{-2}$ with an intensity determined by the data for $E \leq 100 \mathrm{MeV}$. Whereas in the top panel, i.e. without dispersion, the $\mathrm{GeV}$ excess can be clearly seen, the bottom panel proves that a dispersion with $\sigma=0.25$ can in fact explain the data and remove the $\mathrm{GeV}$ excess.

(Ferrari et al. 1996b; Ranft \& Roesler 1994; Möhring et al. 1993; Roesler et al. 1998).

The $\gamma$-ray spectra thus derived can be compared with the observed EGRET spectra from the inner Galaxy. Here we use the data of all viewing periods of phases 1-4, corresponding to observations between 1991 April and 1995 October. From the observed intensity we subtract the extragalactic background (Sreekumar et al. 1998) and the point-spread functions of all sources in the Third EGRET Catalogue (Hartman et al. 1999). The intensity spectrum has additional associated uncertainties, which arise from the subtraction of the extragalactic background and the sources. A systematical error of $10 \%$ is assumed to account for uncertainties in the spark chamber efficiency correction (Esposito et al. 1999).

The pion decay spectra are compared with the intensity of the diffuse Galactic $\gamma$-ray emission from the inner Galaxy in Fig. 1. There the leptonic contribution to the diffuse $\gamma$-ray intensity has been approximated by a simple power law $\propto E^{-2}$ with an intensity $E^{2} I_{l}=$ $0.01 \mathrm{MeV} / \mathrm{cm}^{2} / \mathrm{s} / \mathrm{sr}$, adjusted to reproduce the data for $E \leq 100 \mathrm{MeV}$. It can be seen that a dispersion in the index of the cosmic ray source spectra of $\sigma \simeq 0.25$ is sufficient to explain the observed intensity spectrum and remove the $\mathrm{GeV}$ excess. We can therefore conclude that if dispersion is to be made responsible for the $\mathrm{GeV}$ excess, it must be at a level of $\sigma \simeq 0.25$.

\section{Compatibility with upper limits at higher gamma ray energies}

A dispersion in the cosmic ray source spectra - and thus in the cosmic ray flux throughout the Galaxy - has impact also on the resultant $\gamma$-ray spectra at higher energies, both for the diffuse emission and for individual supernova remnants. In this section we will investigate whether a spectral dispersion of $\sigma=0.25$ is compatible with existing upper limits for $\gamma$-ray emission in the $\mathrm{TeV}$ energy range.

To date, observations of $\mathrm{TeV} \gamma$-ray emission from individual SNR have yielded only a few detections, one of which (Cas A, Aharonian et al. 2001a) is presumably (Atoyan et al. 2000) and the others are clearly caused by leptonic emission. Although deep surveys have been performed, no hadronic $\mathrm{TeV} \gamma$-ray emission has been unambiguously detected from SNR (Buckley et al. 1998). These observational results have been a severe constraint for simple and conventional models of cosmic ray acceleration in SNR. If there were a dispersion in the cosmic ray spectra in SNR, some, i.e. those with a hard spectrum, would be expected to be very prominent $\mathrm{TeV}$ sources. Therefore the observational constraints are even more severe if there is a noteworthy dispersion in the particle spectra.

However, these constraints rely on the assumption that the power law spectra of cosmic rays in the SNR persist to very high energies like $10^{14}-10^{15} \mathrm{eV}$. This may not be a valid assumption; in fact more detailed models of particle acceleration including non-linear effects indicate that the resultant particle spectra may show cut-offs at energies much smaller than $10^{14} \mathrm{eV}$ (Baring et al. 1999). There is also observational evidence that SNR do not produce straight power laws up to $10^{15} \mathrm{eV}$. For all SNR the X-ray flux, synchrotron or not, is less than the extrapolated radio synchrotron spectrum. Since many of the sources, in particular the five historical remnants, are too young for the electron spectra to be limited by energy losses, acceleration cut-offs must occur at electron energies of $100 \mathrm{TeV}$ or less (Reynolds \& Keohane 1999). At these energies the acceleration process should operate similarly for electrons and ions, and similar cut-offs must be expected for the cosmic ray nuclei, which would be intrinsic to the actual acceleration process. Once a remnant is in the Sedov phase, the maximum energy of accelerated particles rises very little. Cas A is probably still in the free expansion phase, but proper-motion measurements of Kepler, Tycho and SN1006 indicate that these objects are well in the Sedov dynamics (Moffett et al. 1993). These remnants are 
therefore unlikely to accelerate cosmic rays to energies higher than $10^{14} \mathrm{eV}$ at any time.

We have made a statistical argument in this paper and therefore may not discuss individual sources, but generally speaking it would seem that a dispersion in the spectra of cosmic rays in SNR is not in conflict with the existing upper limits for $\mathrm{TeV} \gamma$-ray emission, when an upper cut-off in the cosmic ray spectra is taken into account. The same argument applies to the Galactic diffuse $\gamma$-ray emission at $\mathrm{TeV}$ energies as we discuss in somewhat more detail.

The Whipple collaboration has recently published an upper limit for $\gamma$-ray emission at $500 \mathrm{GeV}$ from the Galactic plane, based on observations in a small area in the plane defined by $38.5^{\circ} \leq l \leq 41.5^{\circ}$ and $-2^{\circ} \leq b \leq 2^{\circ}$ (LeBohec et al. 2000). An upper limit for $\gamma$-ray emission at $1 \mathrm{TeV}$ from the Galactic plane $\left(38^{\circ} \leq l \leq 43^{\circ}\right.$, $-2^{\circ} \leq b \leq 2^{\circ}$ ) has been reported by the HEGRA collaboration (Aharonian et al. 2001b). Together with the $\gamma$-ray intensity in the $\mathrm{GeV}$ range these observations constrain models of the $\mathrm{GeV}$ excess. Composite spectra of diffuse Galactic $\gamma$-ray emission from $1 \mathrm{GeV}$ to $500 \mathrm{GeV}$ can be compiled, however, they are subject to systematic uncertainties for a number of reasons. The numerical value of this upper limit at $500 \mathrm{GeV}$ depends to some extent on the $\gamma$-ray spectrum itself. Also the observed portion of sky is so small that the finite extent of the EGRET point-spread function becomes non-negligible. To derive such a composite spectrum of diffuse $\gamma$-ray emission we will therefore use EGRET data for energies above $1 \mathrm{GeV}$ only, for which the $68 \%$ containment radius is $\lesssim 1.7^{\circ}$. To account for the effect of the point-spread function and to increase the number of counts, we will also derive the EGRET intensity spectrum from an area slightly larger than that observed with Whipple, that is $37^{\circ} \leq l \leq 43^{\circ}$ and $-2.5^{\circ} \leq b \leq 2.5^{\circ}$. When comparing the $\pi^{0}$-decay spectrum with the composite spectrum of diffuse Galactic $\gamma$-ray emission, it may be sufficient to use an analytical approximation for the differential pion production cross section, because the uncertainty thus imposed is probably not larger than the systematic uncertainty in the data, but the procedure is substantially simplified. Our analytical approximation for the calculation of the pion source spectra is described in Appendix A.

Given the $\gamma$-ray intensity at $\mathrm{GeV}$ energies, the model intensity at $500 \mathrm{GeV}$ depends on a) the mean proton spectral index (fixed to $\langle s\rangle=2.7$ in this study), b) a possible dispersion in the spectral indices of the cosmic ray sources ( $\sigma=0.25$ is required to account for the $\mathrm{GeV}$ excess), and c) the limiting energy to which the power law spectra of the cosmic ray sources persist. Since we want to investigate whether or not the required dispersion $\sigma=0.25$ is compatible with the Whipple upper limit, the only number which we may vary is the high energy cut-off in the proton spectrum. Therefore the question is: what is the high energy cut-off $\gamma_{\max }$ required to satisfy the Whipple upper limit at $500 \mathrm{GeV}$, given the observed GeV intensity, $<s>=2.7$, and $\sigma=0.25$. Then we may ask whether or not $\gamma_{\max }$ thus derived is reasonable in view of the cosmic ray

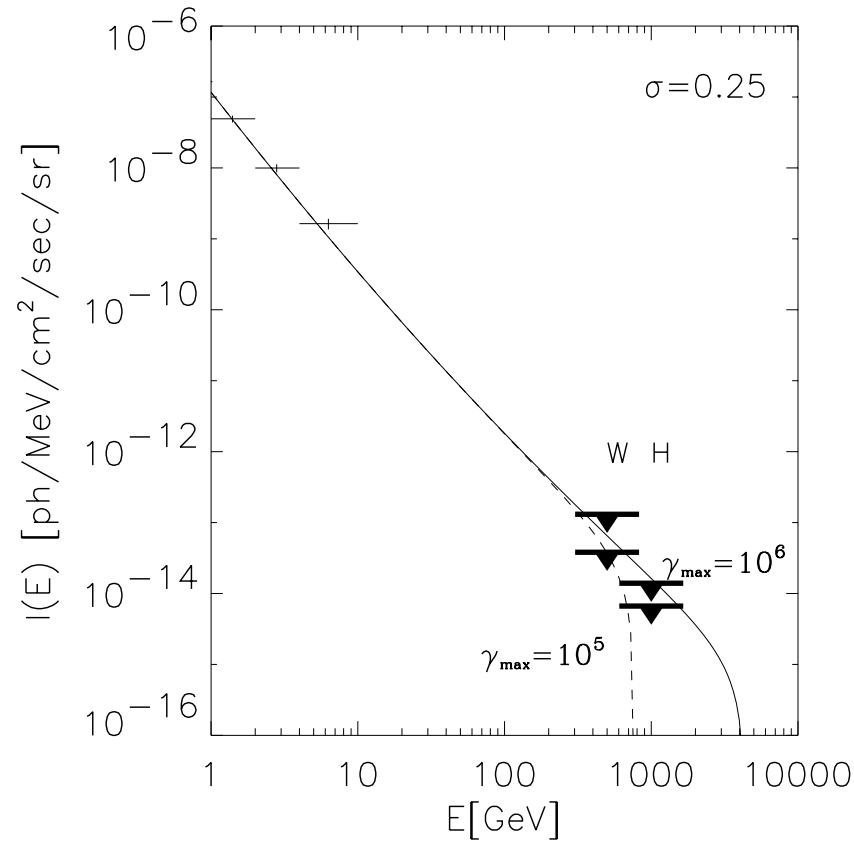

Fig. 2. Composite spectrum between $1 \mathrm{GeV}$ and $1 \mathrm{TeV}$ of the diffuse Galactic $\gamma$-ray emission from the Galactic plane at $l \approx 40^{\circ}$ based on EGRET, Whipple and HEGRA (Aharonian et al. 2001b) data. The upper limit at $500 \mathrm{GeV}$ (Whipple), which is at the $99.9 \%$ confidence level, depends on the $\gamma$-ray spectrum. The lower mark applies for a $\gamma$-ray spectral index $s=2.0$ whereas the upper mark is appropriate for a spectral index $s=2.6$. The limit at $1 \mathrm{TeV}$ (HEGRA), which is a the $99 \%$ confidence level, is plotted for two different ways of background subtraction (see Aharonian et al. 2001b). The data are compared with $\pi^{0}$-decay spectra calculated for a cosmic ray spectrum produced by individual sources with mean spectral index $\langle s\rangle=2.7$ and dispersion $\sigma=0.25$. The solid line refers to a high energy cut-off in the cosmic ray proton spectrum at $\gamma_{\max }=10^{6}$. It should be compared with the lower mark for the upper limit at $500 \mathrm{GeV}$ which it clearly and significantly exceeds. It also exceeds the HEGRA upper limits at $1 \mathrm{TeV}$ for both ways of background subtraction. For $\gamma_{\max }=10^{5}$ the dashed line shows no contradiction with the Whipple and HEGRA data, when compared to the upper mark for the upper limit at $500 \mathrm{GeV}$.

all-particle spectrum and the observed composition near and at the knee.

As can be seen in Fig. 2, a dispersion of $\sigma=0.25$ clearly violates the upper limit at $500 \mathrm{GeV}$ derived by the Whipple team, if $\gamma_{\max }=10^{6}$, but the upper limit is clearly satisfied, if $\gamma_{\max }=10^{5}$. Therefore, if a spectral dispersion exists at the level required to explain the $\mathrm{GeV}$ excess, there must be a high energy cut-off in the cosmic ray source spectra somewhere between $\gamma_{\max }=10^{5}$ and $\gamma_{\max }=10^{6}$.

\section{Discussion}

In this paper we have calculated the high energy diffuse Galactic $\gamma$-ray emission produced by the hadronic component of cosmic rays under the assumption that the interstellar cosmic ray spectrum is a superposition of individual 
power-law spectra with a dispersion in the spectral index as a result of cosmic ray production in SNR. We have shown that a diffuse $\gamma$-ray spectrum thus derived can in fact explain the $\mathrm{GeV}$ excess, provided the dispersion in the individual SNR production spectral indices is $\sigma=0.25$.

We have then investigated under what assumptions a composite cosmic ray spectrum with $\sigma=0.25$ would be compatible with data in the $\mathrm{TeV}$ energy range both for individual SNR and for the diffuse emission. It is found that the high energy data would require the existence of a high energy cut-off in the cosmic ray source spectra at proton energies not higher than somewhere between Lorentz factors $\gamma_{\max }=10^{5}$ and $\gamma_{\max }=10^{6}$.

The hypothesis presented here provides a possible explanation for the $\mathrm{GeV}$ excess, as do other models. It has a number of consequences for observable quantities which can be used to test the viability of the scenario and which we want to discuss in this section.

Is a dispersion of $\sigma=0.25$ compatible with the radio synchrotron spectra of SNR? The following discussion applies only to shell-type SNR which in fact have an average synchrotron power index $\alpha \simeq 0.5$ (Green 2001). This translates to an electron spectral index $s \simeq 2$. For various reasons the uncertainties in the derived radio spectral indices of SNR are often large, but nevertheless some shell-type SNR show spectral indices which are significantly different from 0.5 , either flatter or steeper than the average spectrum. The actual distribution of observed spectral indices results from both an intrinsic dispersion and the observational uncertainties, and it is not a trivial task to deduce the intrinsic dispersion. We have taken the radio spectral index data for Galactic shell SNR given in Fig. 5 of Green (2001) and have performed a $\chi^{2}$-test to see whether the sample of measurements is consistent with a uniform source spectral index. The answer is clearly "no", the reduced $\chi^{2}$ is around 12 . We have then approximated the effect of a distribution of source spectral indices by adding a dispersion $\sigma_{\alpha}$ to the measurement errors. A reduced $\chi^{2}$ of unity results when the dispersion is $\sigma_{\alpha}=0.10$, then also $\langle\alpha\rangle=0.53 \pm 0.02$. Taking out Cas A, which has a soft spectrum with very small error bars, gives $\sigma_{\alpha}=0.085$ and $\langle\alpha\rangle=0.52 \pm 0.02$. This analysis indicates that a dispersion in the radio spectral indices of shell SNR exists, but only at a level of $\sigma_{\alpha}=0.085-0.1$, corresponding to $\sigma_{s}=0.17-0.2$ in the cosmic ray electron spectra. This falls only marginally short of the $\sigma=0.25$ which is required to explain the $\mathrm{GeV}$ excess. Therefore, given the uncertainties, we conclude that $\sigma=0.25$ is compatible with the radio synchrotron spectra of SNR.

Why don't we observe a dispersion in the local cosmic ray spectrum? Here it is useful to calculate the number of SNR which would actually contribute to the local cosmic ray flux. The local supernova rate is about $S=30 \mathrm{Myr}^{-1} \mathrm{kpc}^{-2}$. In standard cosmic ray diffusion models (e.g. Webber et al. 1992) the range of the cosmic rays is linked to the halo size $H \simeq 3 \mathrm{kpc}$ and the life time is identical to the escape time $\tau \simeq 20 \gamma^{-0.6} \mathrm{Myr}$. The number of SNR contributing to the local cosmic ray (proton) flux is then $N=\pi H^{2} \tau S \simeq 17000 \gamma^{-0.6}$. In other words, while at a proton energy of $50 \mathrm{GeV}$ some 1600 SNR would contribute, at $100 \mathrm{TeV}$ the number would be down to $17 \mathrm{SNR}$. Even if this is a rather rough estimate (using an averaged diffusion coefficient for halo and Galactic disc and neglecting reacceleration), it shows clearly that the number of SNR contributing to the local CR flux becomes small at energies of some $100 \mathrm{TeV}$ in which case the local cosmic ray spectrum could strongly deviate from the average spectrum in Eq. (4). A similar effect affects the cosmic ray electron spectrum at much smaller energies of $100 \mathrm{GeV}$, which is the basis for the inverse Compton models of the $\mathrm{GeV}$ excess (Pohl \& Esposito 1998).

Added to this would be the effect of deviations from power law behaviour in the cosmic ray source. We have found that there must be a cut-off at proton energies not higher than a few hundred TeV. This can be established as sharp cut-offs, possibly with a distribution of cut-off energies, or also in the form of a spectral steepening at somewhat smaller energies, which behaviour is predicted in models of non-linear shock acceleration (e.g. Baring et al. 1999). As a result the average spectrum at higher energies would be softer than what is given in Eq. (4), which would not change the expected GeV $\gamma$-ray spectrum, but the local cosmic ray proton spectrum above $10 \mathrm{TeV}$, which is observed to be slightly softer than that obtained by direct measurements at lower energies (Asakimori et al. 1998; Amenomori et al. 2000). All in all the lack of dispersion in the local cosmic ray proton spectrum does not seem to contradict the hypothesis presented in this paper.

Is the high energy limit in the cosmic ray proton source spectra compatible with the observed all-particle spectrum and the composition near the knee? We have seen that the local cosmic ray spectrum near the knee would be produced by few nearby SNR which would cause a structured spectrum near the knee (Erlykin \& Wolfendale 1997a, 1997b, 1998a, 1998b). We have not investigated the composition and in particular not studied the possibly different source spectra for different species. Therefore we feel not well equipped to discuss the issue of cosmic ray composition near the knee. The fact that a softening in the cosmic ray source spectra is required may indicate a potential problem with the notion that SNR accelerate cosmic rays up to the knee, but a more detailed and careful study will be needed to address this issue.

Since there is observational evidence for a dispersion in the spectral indices of the cosmic ray spectra in SNR, the composite cosmic ray spectrum must be curved, if the cosmic rays are predominantly produced in SNR. We have shown that if the dispersion is as strong as $\sigma=0.25$, its effect on the interstellar cosmic ray spectrum would explain the $\mathrm{GeV}$ excess in the diffuse Galactic $\gamma$-ray spectrum. If the actual dispersion is weaker than this, it would still contribute to the $\mathrm{GeV}$ excess and therefore should not be neglected. There are other viable models for the GeV excess and it may well be that it is a combined effect of 
the spectral dispersion and, e.g., a hard inverse Compton spectrum (Pohl \& Esposito 1998).

\section{Appendix A: Approximative calculation of pion spectra}

We consider production of $\pi^{0} \mathrm{~s}$ by cosmic rays via the reaction $\mathrm{p}+\mathrm{p} \rightarrow \pi^{0}+X$, where $X$ stands for any other particle, as described in Mannheim \& Schlickeiser (1994). The total cross section for pion production is $\sigma_{\mathrm{pp}}^{\pi} \simeq \sigma_{\mathrm{p} \text {,inel }} \simeq$ $3 \times 10^{-26} \mathrm{~cm}^{2}$. For charged pions the mean multiplicity has an energy dependence $\xi \simeq 2 E_{\mathrm{p}}^{1 / 4}(\mathrm{GeV})$ where $E_{\mathrm{p}}$ is in GeV. Due to isospin symmetry $\xi_{\pi^{0}}=\frac{1}{2} \xi_{\pi^{ \pm}}$. Laboratory measurements of the mean pion energy in $\mathrm{p}-\mathrm{p}$ and $\mathrm{p}-\alpha$ interactions indicate that a constant fraction, about $30 \%$, of the incident kinetic energy of protons goes to pion energy. This is in agreement with Fermi's theory of pion production, in which a thermal equilibrium is assumed in the resulting pion cloud and in which the mean Lorentz factor of pions in the laboratory frame (where one of the protons is initially at rest) is given by

$\bar{\gamma}_{\pi} \simeq \gamma_{\mathrm{p}}^{3 / 4}$

where $\gamma_{p}$ denotes the Lorentz factor of the incident proton.

We approximate the differential cross-section by the experimentally measured inclusive cross-section multiplied with a $\delta$-function centered at the mean pion energy (A.1), that is

$$
\begin{aligned}
\sum_{k} \sigma_{k, \mathrm{pp}, \mathrm{p} \alpha}^{\pi^{0}}\left(\gamma_{\pi}, \gamma_{\mathrm{p}}\right) & \simeq \xi \sigma_{\mathrm{pp}, \mathrm{p} \alpha}^{\pi^{0}}\left(\gamma_{\mathrm{p}}\right) \delta\left(\gamma_{\pi}-\bar{\gamma}_{\pi}\right) \\
& \simeq \xi \sigma_{\mathrm{pp}, \mathrm{p} \alpha}^{\pi^{0}}\left(\gamma_{\mathrm{p}}\right) \delta\left(\gamma_{\pi}-\gamma_{\mathrm{p}}^{3 / 4}\right)
\end{aligned}
$$

This $\delta$-function approximation and the use of the same mean pion energy (A.1) in $\mathrm{p}-\mathrm{p}$ and $\mathrm{p}-\alpha$ collisions do not introduce appreciable error, provided the incident proton is highly relativistic so that the scaling relations apply. At energies much higher than $10^{4} \mathrm{GeV}$ Eq. (A.2) overestimates the number of pions, but at these energies $\gamma$-rays are also efficiently produced by kaons, so that the use of Eq. (A.2) will not lead to a significant overestimate of the $\gamma$-ray source function. (Dermers 1986 formula yields an inclusive cross section $20 \%$ smaller than that given by Eq. (A.2), with an average pion energy roughly $50 \%$ higher than given by Eq. (A.2), the energy distribution of secondary pions going up almost to the kinetic energy of the incident proton, overpredicting pions at higher rapidities (Mori 1997). Using Dermers formula, the cut off in the $\gamma$-ray spectrum becomes smoother so the required cut off energy in the CR proton spectrum is smaller than in our conservative calculation.)

For the pion power we then obtain

$$
\begin{aligned}
P\left(\gamma_{\pi}, \gamma\right)= & 1.3 \beta c \gamma_{\pi}\left[n_{\mathrm{HI}}(\boldsymbol{r})+2 n_{\mathrm{H}_{2}}(\boldsymbol{r})\right] \xi \sigma_{\mathrm{pp}}^{\pi}(\gamma) \\
& \times \delta\left(\gamma_{\pi}-\gamma^{3 / 4}\right) H\left[\gamma-\gamma_{\mathrm{th}}\right]
\end{aligned}
$$

where the factor 1.30 accounts for the known chemical composition of the interstellar medium. $\gamma_{\text {th }}$ is the hadron threshold Lorentz factor. The pion source function then is

$$
\begin{aligned}
Q_{\pi}\left(\gamma_{\pi} ; \boldsymbol{r}\right)= & \frac{1.26}{\gamma_{\pi} m_{\pi} c^{2}} \int_{1}^{\infty} \mathrm{d} \gamma N(\gamma, \boldsymbol{r}) P_{\pi^{0}}\left(\gamma_{\pi}, \gamma\right) \\
= & \frac{1.64}{m_{\pi} c}\left[n_{\mathrm{HI}}(\boldsymbol{r})+2 n_{\mathrm{H} 2}(\boldsymbol{r})\right] \\
& \times \int_{\gamma_{\mathrm{th}}}^{\infty} \mathrm{d} \gamma N(\gamma, \boldsymbol{r}) \beta \xi \sigma_{\mathrm{pp}}^{\pi^{0}}(\gamma) \delta\left(\gamma_{\pi}-\gamma^{\frac{3}{4}}\right)
\end{aligned}
$$

where the factor 1.26 accounts for the contribution of $\alpha-\mathrm{p}$, $\alpha-\alpha$ collisions and collisions of higher metallicity cosmic rays, and is derived using the ratio of the respective inclusive cross-section to the $\mathrm{p}-\mathrm{p}$ inclusive cross-section and the known elemental composition of the cosmic rays.

For a separable differential number density distribution of cosmic ray hadrons $N(\gamma, \boldsymbol{r})=N_{0}(\boldsymbol{r}) N(\gamma)$ at position $\boldsymbol{r}=(l, b, r)$ in the Galaxy and a spectrum as given in Eq. (4) with a high energy cut-off at $\gamma_{\max }$ we obtain for relativistic particles $(\beta \simeq 1)$

$$
\begin{aligned}
Q_{\pi}\left(\gamma_{\pi} ; \boldsymbol{r}\right)= & \frac{2.2 m_{\mathrm{p}}}{m_{\pi}} N_{0}(\boldsymbol{r})\left[n_{\mathrm{HI}}(\boldsymbol{r})+2 n_{\mathrm{H}_{2}}(\boldsymbol{r})\right] \gamma_{\pi}^{1 / 3} \\
& \times \xi \sigma_{\mathrm{pp}}^{\pi^{0}}\left(\gamma_{\pi}^{4 / 3}\right)\left(\gamma_{\pi}^{8 / 3}-1\right)^{-\frac{s s}{2}+\frac{\sigma^{2}}{8}} \ln \left(\gamma_{\pi}^{8 / 3}-1\right) \\
& \text { for } 1 \ll \gamma_{\pi} \leq \gamma_{\max }^{3 / 4}
\end{aligned}
$$

so that with the assumptions made above, the pion source function (A.5) reduces to

$$
\begin{aligned}
Q_{\pi}\left(\gamma_{\pi} ; \boldsymbol{r}\right) \simeq & \frac{6.6 \times 10^{-26} m_{\mathrm{p}}}{m_{\pi}} N_{0}(\boldsymbol{r})\left[n_{\mathrm{HI}}(\boldsymbol{r})+2 n_{\mathrm{H}_{2}}(\boldsymbol{r})\right] \\
& \times \gamma_{\pi}^{\frac{2-4<s>}{3}+\frac{8 \sigma^{2}}{9} \ln \left(\gamma_{\pi}\right)} \\
& \text { for } 1 \ll \gamma_{\pi} \leq \gamma_{\max }^{3 / 4} .
\end{aligned}
$$

Equation (A.6) can then be used to calculate the $\gamma$-ray source function in Eq. (7).

Acknowledgements. Partial support by the Bundesministerium für Bildung und Forschung through the DLR, grant 50 OR 0006, is gratefully acknowledged.

\section{References}

Abramowitz, M., \& Stegun, I. A. 1972, Handbook of Mathematical Functions, National Bureau of Standards (Washington)

Aharonian, F., Akhperjanian, A., Barrio, J., et al. 2001a, A\&A, 370,112

Aharonian, F., Akhperjanian, A., Barrio, J., et al. 2001b, A\&A, 375,1008

Allen, G. E., Keohane, J. W., Gotthelf, E. V., et al. 1997, ApJ, 487, L97

Amenomori, M., Ayabe, S., Caidong, et al. 2000, Phys. Rev. D, 62, 112002

Asakimori, K., Burnett, T. H., Cherry, M. L., et al. 1998, ApJ, 502,278

Atoyan, A. M., Aharonian, F. A., Tuffs, R. J., \& Völk, H. J. 2000, A\&A, 355, 211

Baring, M. G., Ellison, D. C., Reynolds, S. J., Grenier, I. A., \& Goret, P. 1999, ApJ, 513, 311

Blandford, R. D., \& Eichler, D. 1987, Phys. Rep., 154, 1 
Borkowski, K. J., Rho, J., Reynolds, S. P., \& Dyer, K. K. 2001, ApJ, 550, 334

Brecher, K., \& Burbidge, G. R. 1972, ApJ, 174, 253

Buckley, J. H., Akerlof, C. W., Carter-Lewis, D. A., et al. 1998, A\&A 329, 639

Capella, A., Sukhatme, U., Tan, C.-I., \& Trân Thanh Vân, J. 1994, Phys. Rep., 236, 227

Clark, D. H., \& Caswell, J. L. 1976, MNRAS, 174, 267

Dermer, C. D. 1986, A\&A, 157, 223

Engel, R., Ranft, J., \& Roesler, S. 1997, Phys. Rev. D, 55, 6957

Erlykin, A. D., \& Wolfendale, A. W. 1997, APh, 7, 1

Erlykin, A. D., \& Wolfendale, A. W. 1997, APh, 7, 203

Erlykin, A. D., \& Wolfendale, A. W. 1998, APh, 8, 265

Erlykin, A. D., \& Wolfendale, A. W. 1998, APh, 8, 283

Esposito, J. A., Bertsch, D. L., Chen, A. W., et al. 1999, ApJS, 123,203

Ferrari, A., Sala, P. R., Ranft, J., \& Roesler, S. 1996a, Z. Phys. C, 70, 413

Ferrari, A., Sala, P. R., Ranft, J., \& Roesler, S. 1996b, Z. Phys. C, 71,75

Green, D. A. 2001, in High energy gamma-ray astronomy, ed. F. A. Aharonian, \& H. J. Völk, AIP Conf. Proc., 558, 59

Hartman, R. C., Bertsch, D. L., Bloom, S. D., et al. 1999, ApJS, 123, 79

Hunter, S. D., Bertsch, D. L., Catelli, J. R., et al. 1997, ApJ, 481, 205

Koyama, K., Kinugasa, K., Matsuzaki, K., et al. 1997, PASJ, 49, L7
Koyama, K., Petre, R., Gotthelf, E. V., et al. 1995, Nature, 378,255

LeBohec, S., Bond, I. H., Bradbury, S. M., et al. 2000, ApJ, 539,209

Mannheim, K., \& Schlickeiser, R. 1994, A\&A, 286, 983

Milne, D. K. 1979, Austr. J. Phys., 32, 83

Moffett, D. A., Goss, W. M., \& Reynolds, S. P. 1993, AJ, 106 , 1566

Möhring, H.-J., \& Ranft, J. 1991, Z. Phys. C, 52, 643

Möhring, H.-J., Ranft, J., Merino, C., \& Pajares, C. 1993, Phys. Rev. D, 47, 4142

Mori, M. 1997, ApJ, 478, 225

Muraishi, H., Tanimori, T., Yanagita, S., et al. 2000, A\&A, 354, L57

Pohl, M., \& Esposito, J. 1998, ApJ, 507, 327

Ranft, J., \& Roesler, S. 1994, Z. Phys. C, 62, 329

Ranft, J., Capella, A., \& Trân Thanh Vân, J. 1994, Phys. Lett. B, 320, 346

Reynolds, S. P., \& Keohane, J. W. 1999, ApJ, 525, 368

Roesler, S., Engel, R., \& Ranft, J. 1998, Phys. Rev. D, 57, 2889

Slane, P., Gaensler, B. M., Dame, T. M., et al. 1999, ApJ, 525, 357

Sreekumar, P., Bertsch, D. L., Dingus, B. L., et al. 1998, ApJ, 494,523

Stecker, F. W. 1973, ApJ, 185, 499

Tanimori, T., Hayami, Y, Kamei, S., et al. 1998, ApJ, 497, L25

Webber, W. R., Lee, M. A., \& Gupta, M. 1992, ApJ, 390, 96 